Supporting Information

\title{
Three-Dimensional Ni Foam Supported CoO Nanoparticles/N-Doped Carbon Multilayer Nanocomposite Electrode for Oxygen Evolution
}

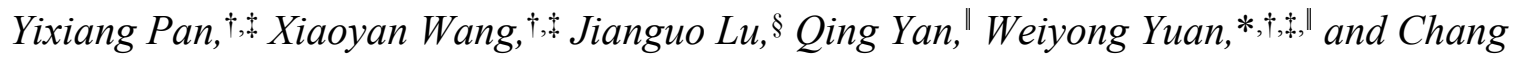
Ming $L i^{\dagger}, *$

${ }^{\dagger}$ Institute for Clean Energy and Advanced Materials, School of Materials \& Energy, Southwest University, Chongqing 400715, China

¥Chongqing Key Laboratory for Advanced Materials and Technologies of Clean Energies, Chongqing 400715, China

\$State Key Laboratory of Silicon Materials, School of Materials Science and Engineering, Zhejiang University, Hangzhou 310027, China

"Ningbo Research Institute, Zhejiang University, Ningbo 315100, China

*Corresponding author.E-mail address: yuanweiyong@swu.edu.cn

\section{Contents}

1. The performance of representative OER catalysts measured in $0.1 \mathrm{M} \mathrm{KOH}$ (Table S1).

2. XRD pattern (A) and FESEM image (B) of NF@CoO NSA (Figure S1).

3. Charging current density differences of NF@(CoO NP/NC $)_{2}$ and NF@CoO NSA at a potential of $1.05 \mathrm{~V}$ plotted against scan rates (Figure S2). 
Table S1. The performance of representative OER catalysts measured in $0.1 \mathrm{M} \mathrm{KOH}$.

\begin{tabular}{|c|c|c|c|}
\hline Catalyst & $\begin{array}{l}\text { Overpotential at } \\
10.0 \mathrm{~mA} \mathrm{~cm}^{-2}(\mathrm{mV})\end{array}$ & $\begin{array}{l}\text { Tafel slope } \\
\left(\mathrm{mV} \mathrm{dec}^{-1}\right)\end{array}$ & Ref. \\
\hline $\mathrm{RuO}_{2}$ & 355 & 63 & 1 \\
\hline $\mathrm{IrO}_{2} / \mathrm{C}$ & 370 & N.A. & 2 \\
\hline $\mathrm{IrO}_{2} / \mathrm{CNT}$ & 360 & 90 & 3 \\
\hline $\mathrm{IrO}_{2}$ & 540 & 130 & 4 \\
\hline $\mathrm{RuO}_{2}$ & 420 & 72 & 5 \\
\hline $\mathrm{RuO}_{2} / \mathrm{CC}$ & 400 & 112.9 & 6 \\
\hline $20 \mathrm{wt} \% \mathrm{Ir} / \mathrm{C}$ & 409 & 126 & 7 \\
\hline $\mathrm{IrO}_{2}$ & 400 & 85.5 & 8 \\
\hline $20 \mathrm{wt} \% \mathrm{Ru} / \mathrm{C}$ & 390 & N.A. & 9 \\
\hline FeNi@NGE/NC & 372 & 55.2 & 10 \\
\hline $\mathrm{RuO}_{2}$ & 355 & 63 & 1 \\
\hline Oxidized carbon cloth & 477 & 82 & 11 \\
\hline $\mathrm{Ti}_{3} \mathrm{C}_{2} \mathrm{~T}_{\mathrm{x}}-\mathrm{CoBDC}$ hybrid & 410 & 48.2 & 12 \\
\hline $\operatorname{Sr}\left(\mathrm{Co}_{0.8} \mathrm{Fe}_{0.2}\right)_{0.7} \mathrm{~B}_{0.3} \mathrm{O}_{3-\delta}$ & 340 & 58 & 13 \\
\hline $3 \mathrm{D}-\mathrm{C}_{3} \mathrm{~N}_{4}$ NS-CNT & 370 & 83 & 3 \\
\hline NF@CoOLNSA@G & 330 & 79 & 14 \\
\hline $\mathrm{NF} @(\mathrm{PEI} / \mathrm{RGO})_{2}$ & 322 & 78 & 15 \\
\hline MIL-88A/Ni(OH $)_{2}-\mathrm{CC}$ & 352 & 46.1 & 6 \\
\hline PDDA@CNT membrane & 370 & 76 & 16 \\
\hline PANI-FeCo/MWCNT & 440 & 55 & 4 \\
\hline $\mathrm{NiCo}_{2} \mathrm{~S}_{4}$ hollow spheres & 400 & 93.7 & 8 \\
\hline $\mathrm{CuO} @ \mathrm{CoFeO}_{\mathrm{x}}$ & 360 & 57.8 & 17 \\
\hline $\begin{array}{c}\text { amorphous } \mathrm{MoS}_{\mathrm{x}} \text {-encapsulated } \\
\mathrm{Co}(\mathrm{OH})_{2} \text { nanosheets }\end{array}$ & 350 & 65.4 & 18 \\
\hline SCF- 800 & 327 & 62 & 19 \\
\hline rGO@CoNiO & 320 & 45.0 & 20 \\
\hline RP/P-LSCF & 324 & 58 & 1 \\
\hline $\mathrm{NF} @(\mathrm{CoO} \mathrm{NP} / \mathrm{NC})_{2}$ & 318 & 46.1 & This work \\
\hline
\end{tabular}



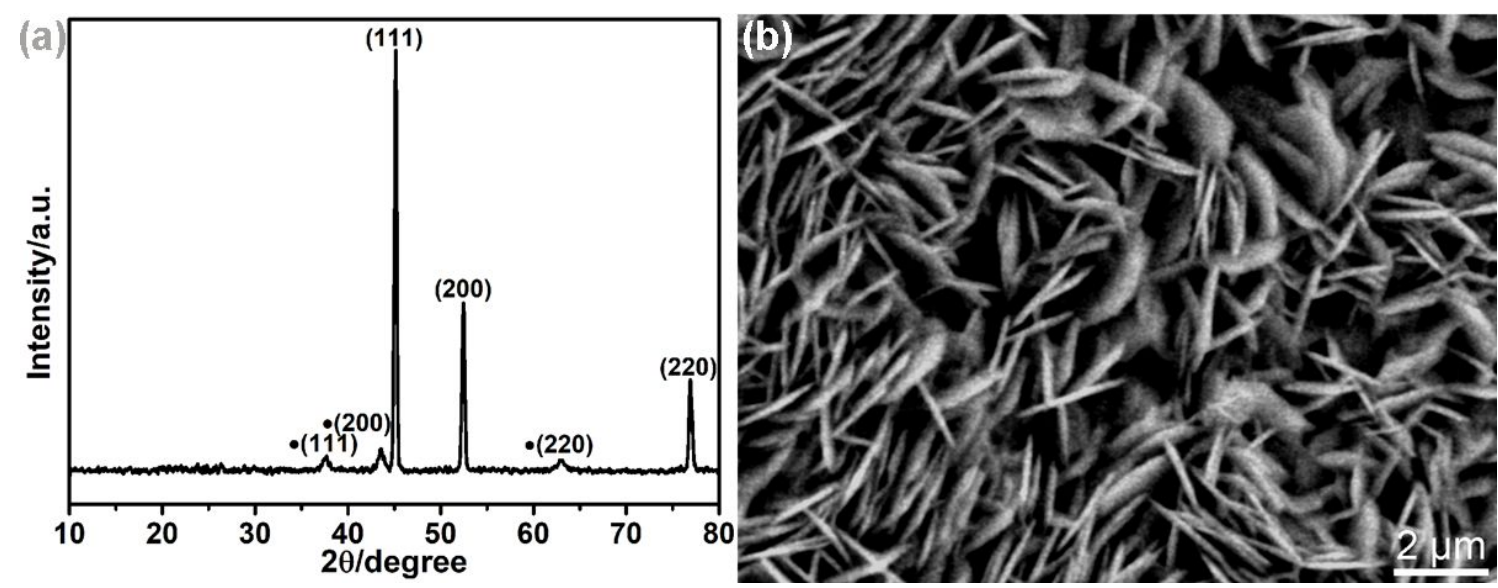

Figure S1. XRD pattern (a) and FESEM image (b) of NF@CoO NSA. The XRD pattern shows the characteristic peaks of cubic CoO (JCPDS No. 43-1004) (those with •) and of cubic Ni (JCPDS No. 01-1258) (those without $\bullet$ ), which are from the Ni foam.

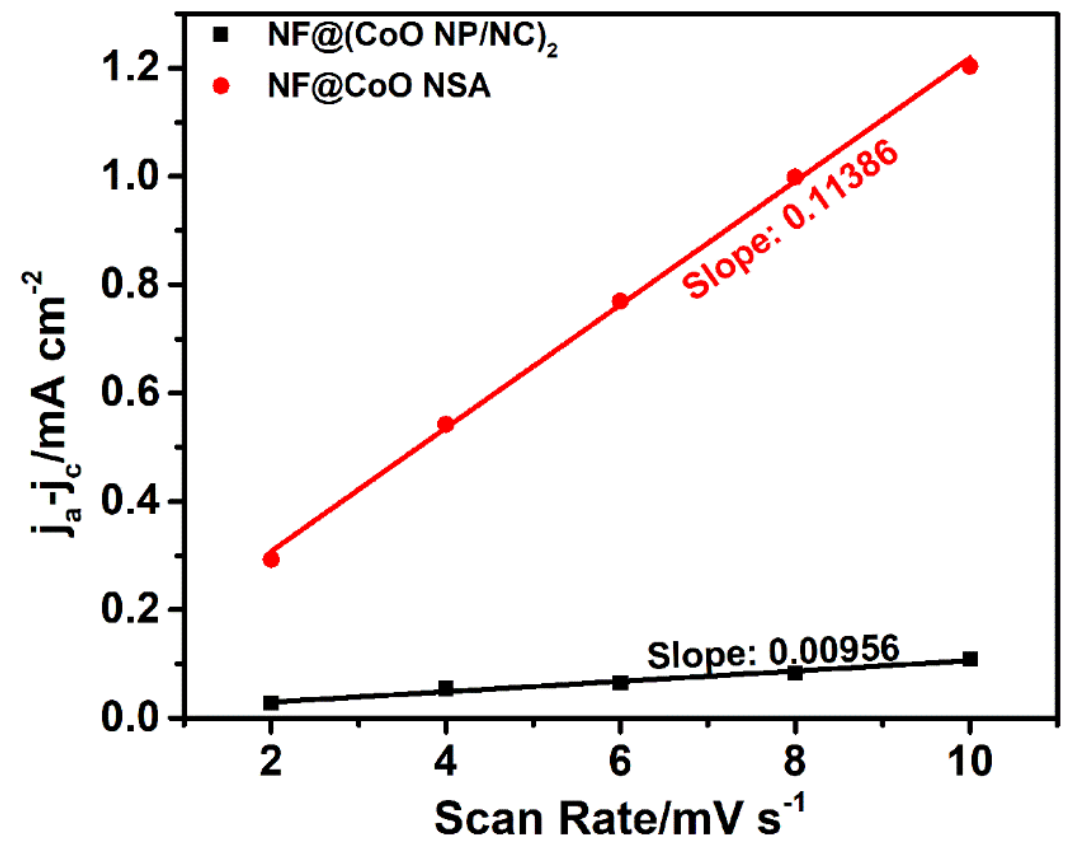

Figure S2. Charging current density differences of $\mathrm{NF} @(\mathrm{CoO} \mathrm{NP} / \mathrm{NC})_{2}$ and $\mathrm{NF} @ \mathrm{CoO}$ NSA at a potential of $1.05 \mathrm{~V}$ plotted against scan rates. These scan rates are chosen because both samples show excellent linearity in this scan rate region. 


\section{References}

[1] Zhu, Y.; Lin, Q.; Hu, Z.; Chen, Y.; Yin, Y.; Tahini, H. A.; Lin, H.-J.; Chen, C.-T.; Zhang, X.; Shao, Z.; Wang, H. Self-Assembled Ruddlesden-Popper/Perovskite Hybrid with Lattice-Oxygen Activation as a Superior Oxygen Evolution Electrocatalyst. Small 2020, 16, 2001204.

[2] Zhao, Y.; Nakamura, R.; Kamiya, K.;Nakanishi, S.; Hashimoto, K. NitrogenDoped Carbon Nanomaterials as Non-Metal Electrocatalysts for Water Oxidation. Nat. Commun. 2013, 4, 2390.

[3] Ma, T. Y.; Dai, S.; Jaroniec, M.; Qiao, S. Z. Graphitic Carbon Nitride NanosheetCarbon Nanotube Three-Dimensional Porous Composites as High-Performance Oxygen Evolution Electrocatalysts. Angew. Chem. Int. Ed. 2014, 53, 7281-7285.

[4] Zhao, C.; Jin, Y.; Du, X.; Du, W. In situ Prepared Amorphous FeCoOPolyaniline/Multiwalled Carbon Nanotube Nanohybrids as Efficient Oxygen Evolution Catalysts for Rechargeable Zn-air Batteries. J. Power Sources 2018, 399, 337-342.

[5] Li, L.; Tian, T.; Jiang, J.; Ai, L. Hierarchically Porous $\mathrm{Co}_{3} \mathrm{O}_{4}$ Architectures with Honeycomb-like Structures for Efficient Oxygen Generation from Electrochemical Water Splitting. J. Power Sources 2015, 294, 103-111.

[6] Qian, Z.; Wang, K.; Shi, K.; Fu, Z.; Mai, Z.; Wang, X.; Tang, Z.; Tian, Y. Interfacial Electron Transfer of Heterostructured MIL-88A/Ni(OH $)_{2}$ Enhances the Oxygen Evolution Reaction in Alkaline Solutions. J. Mater. Chem. A 2020, 8, 3311-3321.

[7] Sa, Y. J.; Kwon, K.; Cheon, J. Y.; Kleitz, F.; Joo, S. H. Ordered Mesoporous $\mathrm{Co}_{3} \mathrm{O}_{4}$ Spinels as Stable, Bifunctional, Noble Metal-Free Oxygen Electrocatalysts. $J$. Mater. Chem. A 2013, 1, 9992-10001.

[8] Feng, X.; Jiao, Q.; Cui, H.; Yin, M.; Li, Q.; Zhao, Y.; Li, H.; Zhou, W.; Feng, C. One-Pot Synthesis of $\mathrm{NiCo}_{2} \mathrm{~S}_{4}$ Hollow Spheres via Sequential Ion-Exchange as an Enhanced Oxygen Bifunctional Electrocatalyst in Alkaline Solution. ACS Appl. Mater. Interfaces 2018, 10, 29521-29531.

[9] Gorlin, Y.; Jaramillo, T. F. A Bifunctional Nonprecious Metal Catalyst for Oxygen Reduction and Water Oxidation. J. Am. Chem. Soc. 2010, 132, 13612-13614.

[10] Shah, S. A.; Ji, Z.; Shen, X.; Yue, X.; Zhu, G.; Xu, K.;Yuan, A.; Ullah, N.; Zhu, J.; Song, P.; Li, X. Thermal Synthesis of FeNi@Nitrogen-Doped Graphene Dispersed on Nitrogen-Doped Carbon Matrix as an Excellent Electrocatalyst for Oxygen Evolution Reaction. ACS Appl. Energy Mater. 2019, 2, 4075-4083.

[11] Cheng, N.; Liu, Q.; Tian, J.; Xue, Y.; Asiri, A. M.; Jiang, H.; He, Y.; Sun, X. Acidically Oxidized Carbon Cloth: a Novel Metal-Free Oxygen Evolution Electrode with High Catalytic Activity. Chem. Commun. 2015, 51, 1616-1619.

[12] Zhao, L.; Dong, B.; Li, S.; Zhou, L.; Lai, L.; Wang, Z.; Zhao, S.; Han, M.; Gao, K.; Lu, M.; Xie, X.; Chen, B.; Liu, Z.; Wang, X.; Zhang, H.; Li, H.; Liu, J.; Zhang, H.; Huang, X.; Huang, W. Interdiffusion Reaction-Assisted Hybridization of TwoDimensional Metal-Organic Frameworks and $\mathrm{Ti}_{3} \mathrm{C}_{2} \mathrm{~T}_{\mathrm{x}}$ Nanosheets for Electrocatalytic Oxygen Evolution. ACS Nano 2017, 11, 5800-5807.

[13] She, S.; Zhu, Y.; Chen, Y.; Lu, Q.; Zhou, W.; Shao, Z. Realizing Ultrafast Oxygen Evolution by Introducing Proton Acceptor into Perovskites. Adv. Energy Mater. 2019, 9, 1900429.

[14] Yuan, W.; Zhao, M.; Yuan, J.; Li, C. M. Ni Foam Supported Three-Dimensional 
Vertically Aligned and Networked Layered $\mathrm{CoO}$ Nanosheet/Graphene Hybrid Array as a High-performance Oxygen Evolution Electrode. J. Power Sources 2016, 319, 159-167.

[15] Zhao, M.; Yuan, W.; Li, C. M. Controlled Self-Assembly of Ni Foam Supported Poly(ethyleneimine)/Reduced Graphene Oxide Three-Dimensional Composite Electrodes with Remarkable Synergistic Effects for Efficient Oxygen Evolution. $J$. Mater. Chem. A 2017, 5, 1201-1210.

[16] Mo, C.; Jian, J.; Li, J.; Fang, Z.; Zhao, Z.; Yuan, Z.; Yang, M.; Zhang, Y.; Dai, L.; $\mathrm{Yu}, \mathrm{D}$. Boosting Water Oxidation on Metal-Free Carbon Nanotubes via Directional Interfacial Charge-Transfer Induced by an Adsorbed Polyelectrolyte. Energy Environ. Sci. 2018, 11, 3334-3341.

[17] Chen, G.; Guo, Y.; Miao, S.; Sun, H.; Gu, B.; Li, W.; Zhong, Y.; Zhou, W.; Shao, Z. Constructing Self-Standing and Non-Precious Metal Heterogeneous Nanowire Arrays as High-Performance Oxygen Evolution Electrocatalysts: Beyond the Electronegativity Effect of the Substrate. J. Power Sources 2018, 396, 421-428.

[18] Sun, F.; Li, C.; Li, B.; Lin, Y. Amorphous $\mathrm{MoS}_{\mathrm{x}}$ Developed on $\mathrm{Co}(\mathrm{OH})_{2}$ Nanosheets Generating Efficient Oxygen Evolution Catalysts. J. Mater. Chem. A 2017, 5, 23103-23114.

[19] Lin, Q.; Zhu, Y.; Hu, Z.; Yin, Y.; Lin, H.-J.; Chen, C.-T.; Zhang, X.; Shao, Z.; Wang, H. Boosting the Oxygen Evolution Catalytic Performance of Perovskites via Optimizing Calcination Temperature. J. Mater. Chem. A 2020, 8, 6480-6486.

[20] Li, P.; Zeng, H. C. Sandwich-Like Nanocomposite of $\mathrm{CoNiO}_{x} /$ Reduced Graphene Oxide for Enhanced Electrocatalytic Water Oxidation. Adv. Funct. Mater. 2017, 27,1606325 . 